\title{
Téoros
}

Revue de recherche en tourisme

\section{Du rêve à la réalité}

L'information touristique dans les quotidiens

\section{Pierre Vincent}

Volume 4, numéro 1, mars 1985

L'information touristique

URI : https://id.erudit.org/iderudit/1080735ar

DOI : https://doi.org/10.7202/1080735ar

Aller au sommaire du numéro

Éditeur(s)

Université du Québec à Montréal

ISSN

0712-8657 (imprimé)

1923-2705 (numérique)

Découvrir la revue

\section{Citer cet article}

Vincent, P. (1985). Du rêve à la réalité : l’information touristique dans les quotidiens. Téoros, 4(1), 4-6. https://doi.org/10.7202/1080735ar d'utilisation que vous pouvez consulter en ligne.

https://apropos.erudit.org/fr/usagers/politique-dutilisation/ 


\section{L'information touristique dans les quotidiens}

\section{Du rêve à la réalité}

par Pierre Vincent *

L'information touristique occupe-t-elle une bonne place dans les médias? $\mathrm{Ce}$ type d'information spécialisée répond-il à tous les besoins des lecteurs? L'information touristique québécoise peut-elle être comparée avantageusement à celle que l'on trouve dans les médias à l'étranger?

\section{L'espace occupé}

A la première question, il ne fait pas de doute qu'il faut sans hésiter répondre par l'affirmative: par rapport à bien d'autres secteurs de l'information, les "actualités" touristiques prennent une place considérable dans nos médias. Une place considérable et même, jusqu'à un certain point, démesurée par rapport aux centres d'intérêt et aux préoccupations de l'immense majorité des citoyens.

Point n'est besoin d'être grand clerc pour constater que tous les grands quotidiens, La Presse, The Gazette, Le Journal de Montréal et Le Soleil, y consacrent des cahiers presque entiers dans les éditions du week-end, tandis que Le Devoir y réserve également ses plus belles pages du samedi.

Les patrons de certains quotidiens poussent même leur intére̊t pour le contenu rédactionnel touristique au point d'affecter à cette spécialisation un nombre de journalistes souvent plus élevé que pour bien d'autres secteurs névralgiques de l'information.

Un exemple: à La Presse, pour deux postes de chroniqueurs touristiques permanents, il n'y a qu'un poste de chroniqueur pour couvrir le vaste domaine de l'éducation; qu'un poste pour s'occuper de tout I'univers du travail; qu'un poste pour s'intéresser aux sciences et aux technologies: nouvelles.... Le quotidien de la rue SaintJacques ne fait ainsi que suivre une vieille habitude de journaux nord-américains qui, soit dit en passant, a su résister efficacement à tous les mouvements de compres: sion budgétaires des dernières années.

"Pierre Vincent est joumaliste do La Presse.
Dans les médias électroniques, la crise économique a toutefois quelque peu modifié les habitudes: les émissions touristiques à proprement parler sont disparues. Les secondes de la télévision et les minutes de la radio étant plus précieuses et, surtout, plus coûteuses que les lignes agates, il semble que les annonceurs et les diffuseurs en soient venus à la conclusion que les consommateurs de beaux rêves exotiques ne sont plus aujourd'hui assez nombreux à l'antenne pour justifier une poursuite de dépenses en énergies humaine et financière dans des émissions et des chroniques de voyages. D'autant que tous les spécialistes s'entendent depuis un bon moment pour reconnaître que les consommateurs de voyages finissent toujours invariablement par ẻtre les mëmes, que ce sont des gens qui se fient plus souvent qu'autrement à leur experience personnelle pour aller négocier un forfait de vacances au comptoir d'une compagnie aérienne ou d'une agence de voyages.

Mais, tout cela étant dit, les évocations aux merveilleuses vacances exotiques $\mathrm{n}$ 'ont jamais cessé de se multiplier dans les médias électroniques, si bien que le contenu touristique $\mathrm{y}$ est toujours présent; pour les marchands de vacances, il est en effet beaucoup plus simple et beaucoup plus pratique de payer quelques jours de vacances à tel morning man ou à telle chroniqueuse, qui se répandront éventuellement en douces effusions à des émissions "prime time", que de commanditer une émission faite par des professionnels de l'information.

\section{Des lecteurs satisfaits?}

Enfin brefl, I'information touristique, com= prise dans un sens très large, il va sans dire, occupe une grande place dans les médias du Québec. Mais, de quelle information touristique s'agit-il? Délicate question, $\mathrm{s}^{\prime} \mathrm{i}$ en est une! Question dont la réponse vaut certainement une bonne partie de la cagnote de la dernière loterie. Oublions pour simplifier, le fait que l'information touristique pourrait s'adresser a un plus vaste public que maintenant et contentons-nous de voir à qui elle s'adresse dans sa facture actuelle. Et entendons-nous pour admet- tre qu'il y a aujourd'hui quatre grands types de lecteurs de pages touristiques.

1) Le lecteur qui voyage par procuration. Celui qui n'a pas let quil n'aura peut-être jamais) les moyens de partir au bout du monde. Ou encore qui n'a ni l'imagination ni l'assurance nécessaire pour entreprendre des voyages dans des pays oủ vivent des gens trop différents de lui-même.

C'est le lecteur le mieux servi par les chroniqueurs touristiques! Qui, parfois, excellent vraiment dans le genre de papiers expressionnistes sur leurs propres expériences de voyages ou, ả l'occasion, sur celles vécues par des vacanciers rencontrés au hasard de leurs reportages à l'étranger.

J'ai toujours été frappé par la scrupuleuse observance de l'ensemble des chroniqueurs de ce qui apparaît comme règle de base dans la rédaction des articles touristiques: il faut, semble-t-il, toujours ètre positif, insister surtout sur ce qui est beau et "le fun", glisser sur les aspects négatifs d'une destination de vacances et eviter, autant que faire se peut, de décrire les réalités socio-politiques avec lesquelles, pourtant, n'importe quel voyageur le moindrement conscient sera confronté. Pourquoi un critique de cinéma peut-il, s'il le juge à propos, se permettre de démolir une superproduction, qui aura coûté des millions de dollars, et pourquoi les journalistes de tourisme n'écrivent-ils jamais que telle nouvelle destination de vacances concoctée dans les officines des grossistes en voyage ne vaut pas le déplacement?

Du temps où j"étais chroniqueur touristique à plein temps, je me souviens d'avoir dû insister lourdement pour passer à la une d'un cahier de vacances-voyages une photo illustrant éloquemment la misère de villageois sud-africains vivant à l'ombre de sélects clubs réservés aux Blancs. "C̣a n'invite pas tellement les gens à partir en voyage" "m'alléguait-on candidement.

Je ne suis pas prêt d'oublier non plus cette autre anecdote qui démontre bien que les règles du jeu dans ce secteur de l'information obéissent bien plus aux impératif́s des publicitaires qu'à ceux des professionnels 
de l'information. J'avais été invité par Air France et par l'Office du tourisme tunisien à faire un sejour dans ce pays du Magreb. Le but de l'exercice, m'avait-on laissé entendre avant le départ, consiste à faire valoir aux Québécois que la Tunisie peut être une destination-soleil aussi agréable que la Floride ou le Mexique en hiver. Sans compter, ajoutait-on, coup d'oeil complice en coin, que la Tunisie est un pays français oủ un Québécois francophone peut circuler comme chez lui.

Or, apres une semaine de sejour, aprẻs m'étre promené aux quatre coins du pays, après avoir rencontré de nombreux coopérants canadiens en poste là-bas et après avoir vécu diverses expériences avec des dizaines et des dizaines de Tunisiens, j'en étais venu a la conclusion que la Tunisie est un pays fascinant, extraordinairement fascinant, mais qu'il ne fallait pas croire que le climat tunisien en hiver est aussi invitant que celui du Mexique ou des Antilles. En outre, je rapportais que tous les Tunisiens ne sont pas toujours en mesure de venir en aide a un touriste unilingue françis, le pays s'etant affranchi de la France depuis une bonne génération et bien des Tunisiens se sentant aujourd'hui plus près de la culture arabe que de celle de M. Malraux. J'écrivis tout cela, ainsi que tout le bien que je pensais de la Tunisie, dans une série d"articles dont la publication s'échelonna sur trois ou quatre semaines.

Cela déplut souverainement ă ces messieurs de l'Office du tourisme tunisien, du consulat et mame de l'ambassade, qui firent pression sur la direction de mon journal pour que cesse sur le champ ce qu'ils considéraient comme une "campagne de

\title{
TAGANGWS
}

\section{L'incitation au départ}

\section{Enquête auprès des lecteurs du cahier Vacances-Voyage du journal La Presse}

\author{
par Renée Asselin et Louis Pedneault
}

Dans le cadre de cette recherche, nous avons tracé le profil socio-économique des lecteurs du cahier Vacances-Voyage; les principales caractéristiques des lecteurs sont les suivantes:

- composés d'un peu plus d'hommes que de femmes:

- l'âge moyen est de 35 ans"

- un niveau de scolarisation relativement élevé;

- le revenu familial se situant, en moyenne, dans la fourchette $\$ 20,000 / \$ 40,000$.

Les habitudes de voyages des répondants ont étét étudiées; à ce sujet nous pouvons remarquer:

- que les lecteurs favorisent les Etats-Unis comme principale destination;

- que la détente et le soleil demeurent le but du départ:

- que les lecteurs font, en moyenne, un voyage par année d'une durée de deux semaines et dans $50 \%$ des cas, accom. pagnés de leur conjoint(e):

- que la principale source d'information se résume aux parents et amis.

Il semble que la façon de voyager que propose le cahier Vacances-Voyage correspond au profil-type de ces lecteurs.

Le cahier propose principalement des destinations de masse et des circuits touristiques, conçus pour des voyages autonomes et le plus souvent comtemplatifs. Le profil du lecteur et de ses habitudes de voyage. que nous avons dressé auparavant correspond effectivement a ces formes de vovage.

En ce qui concerne le cahier VacancesVoyage proprement dit, les principaux résultats obtenus sont:

- que $83 \%$ des lecteurs du journal La Presse consultent la section Vacances: Voyage:

- que la majorité d'entre eux sont attirés par la lecture des articles mais que les destinations québécoises ne sont pas suffisamment abordees:

- que la grande majorité d'entre eux dit n'avoir jamais été influencée par leur lecture quant aux choix d'une destination:

- que les destinations traitées sont suffisamment variées;

- que le fait d'accompagner les articles de photos couleurs peut effectivement attirer davantage le lecteur.

De façon générale, les gens sont satisfaits du cahier Vacances-Voyage. IIs trouvent que les destinations traitées sont assez variées, par contre, ils aimeraient retrouver de plus amples informations en ce qui concerne les prix et les choses à faire ou à ne pas faire. Les gens sont également satisfaits de la publicite qu'il contient. IIs affirment que le contenu publicitaire peut leur servir de source d'information et de guide d'achat dans la planification d'un éventuel départ.

L'enquête cherchait à confirmer que la lecture du cahier Vacances-Voyage incite l'usager au départ et influence son choix d'itinéraire. II fut confirmé que la consultation des nouvelles et de la publicité est effectivement stimulante. Mais on ne se dit que peu influencé par le choix des destinations proposées. Le lecteur se reconnait une autonomie, une liberté de choix dans ses intentions de déplacements tout en admettant volontiers que son désir de partir s'amplifie a la lecturé du cahier Vacances-Voyage.

Plus on lit, plus on veut partir mais on ne veut pas se faire dire oủ aller. On veut choisir. On déplore le manque de détails techniques qui devraient accompagner tout déplacement important mais on reconnait la qualité des articles, de la présentation et de l'incitation au rêve que le cahier procure.

Les gens qui voyagent ne consultent pas ce cahier pour choisir une destination particulière, mais plutôt parce que celle-ci leur offre l'opportunité de s'évader et de voyager à longueur d'année...sans se déplacer.

\section{Méthodologie}

Cette enquête a été réalisée sous la direction de Jean Stafford dans le cadre du cours Méthodologie de la recherche en tourisme à l'Université du Québec à Montréal. Les répondants ont été choisis, au hasard systématique, à partir d'un échantillon aléatoire tiré du bottin téléphonique du Grand Montréal. La cueillette des données a été faite entre le 15 novembre et le 15 décembre 1984; 100 entrevues ont été complétées et le taux de réponse est de $35,84 \%$. La marge d'erreur théorique sur les réponses est de $9 \%$ au seuil de confiance de $95 \%$. 
harcelement"r contre leur pays. Mes patrons d'alors ne cédèrent pas au chantage de coupures d'annonces ou de je ne sais trop quoi, mais ils finirent par consentir de dépecher en Tunisie un autre journaliste, qui, de toute évidence, devrait livrer à son retour un message résolument conforme aux désiderata des concepteurs du ministère du Tourisme tunisien. On offrit le voyage, avec "VIP treatment" s'il vous plaît, à un collègue qui n'avait jamais mis les pieds dans un avion de sa vie; il revint de son voyage absolument ravi, le journal publia ses papiers dithyrambiques sans explications, et tout le monde considéra l'incident clos. Sauf moi.

2) Le deuxième lecteur à consulter les pages touristiques des grands quotidiens québécois, c'est celui qui cherche à s'instruire sur les curiosités et les merveilles du monde, à se cultiver. A parfaire sa culture livresque, en attendant sans doute l'occasion de l'approfondir dans des ouvrages plus consistants que les articles de journaux.

Les beautés des pyramides d'Égypte, les aubaines du shopping à Hong Kong, les menaces qui pèsent sur la survie des tortues géantes aux îles Galapagos, les der= nières trouvailles de Disney World, tout sur tel festival d'art gothique en Europe, les splendeurs du parc Kruger en Afrique du Sud, les travaux de restauration d'une cathédrale à Berlin, la description d'un marché aux poissons à Tokyo, tout y passe, avec des titres plus ou moins gros. Et pourquoi pas? Pourquoi les grands journaux populaires ne poursuivraient-ils pas le travail des professeurs d'histoire et de géographie?

Sauf que je me prends parfois à regretter que ce genre d'information, si aisément accessible aux communs des mortels, semble rendre la vie trop facile aux chroniqueurs de tourisme qui ne se sentent plus aussi pressés d'aller quérir d'autres nouvelles. Ainsi, par exemple, pendant les vacances des fêtes, j'ai cherché désespérément dans les pages touristiques des grands journaux des suggestions utiles pour mieux vivre ma québécitude. Tout ce que j'ai trouvé, c'est un grand placard publicitaire énumérant différentes auberges, mais nulle part quelqu'un pour me décrire objectivement les vertus de telle ou telle bonne adresse.

3) Le lecteur à la recherche d'information pertinente sur l'art de bien consommer les produits touristiques est, en fait, celui qui reste le plus sur son appétit. Bien sûr, il n'est pas facile de satisfaire les besoins des consommateurs de vacances. "Même avec la meilleure volonté du monde, je ne peux être allé partout dans. le monde, en revenir la veille et, par conséquent, je ne suis pas capable d'informer

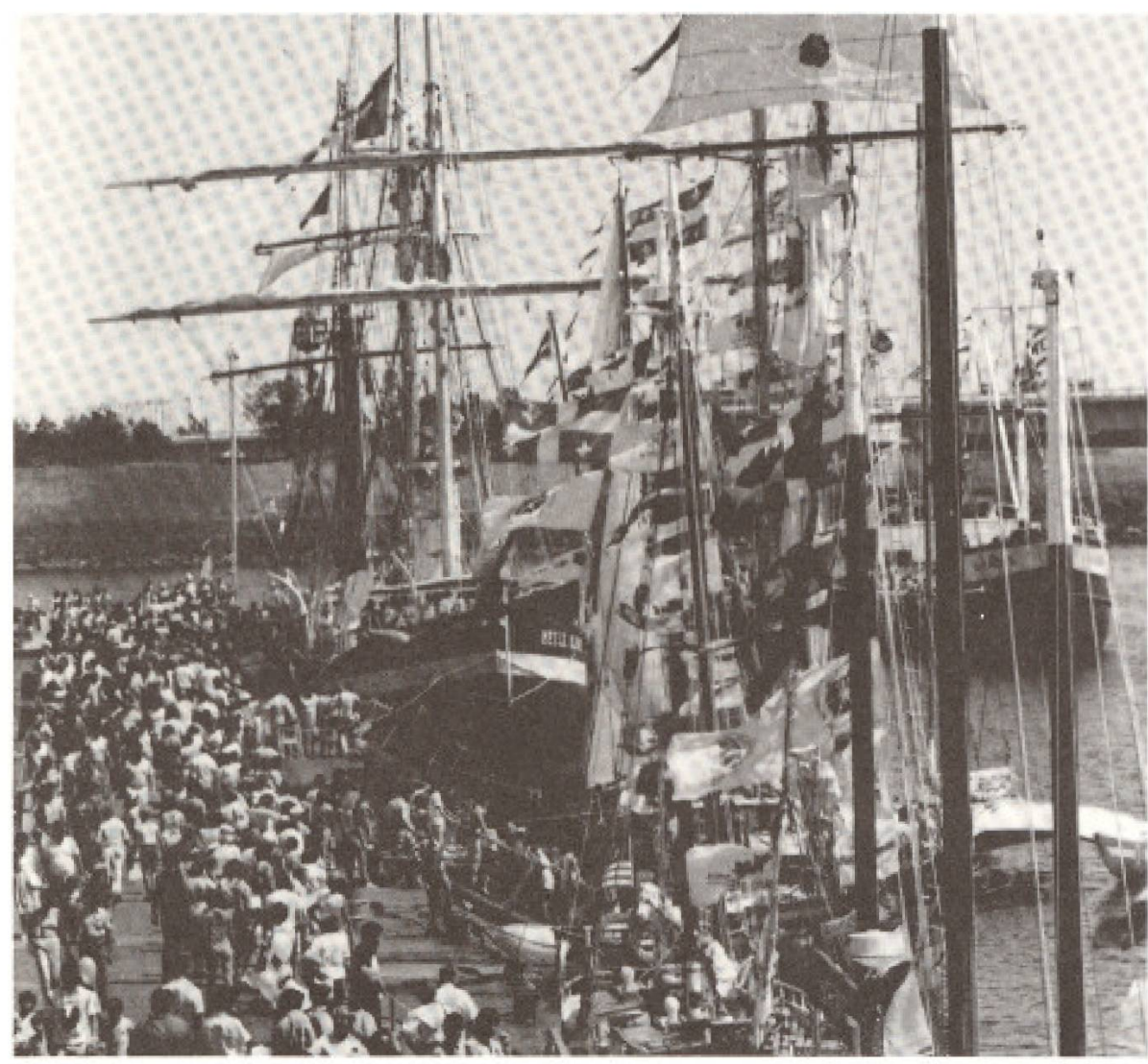

Sí on a dénombré, l'été dernier, plusieurs articles sur la venue des grands voiliers à Québec et à Montréal, il n'est pas toujours facile d'obtenir une information objective sur le Québec dans les pages touristiques des grands quotidiens. mes clients aussi adéquatement que comme si je leur vendais des vêtements" avait I'habitude d'expliquer un agent de voyages.

N'emperche qu'il pourrait $y$ avoir plus de journalisme d'enquête dans les pages touristiques. Cela permettrait à tout le moins aux lecteurs d'être mieux outillés pour porter un jugement sur ce qu'on leur propose. Comment les spécialistes s'y prennent-ils pour mettre Cuba a la mode une année et la République dominicaine l'année suivante? En vertu de quoi modifie-t-on ainsi les modes? En vertu des goûts des clients? Des compromissions des gouvernements?

II faut bien se rendre à l'évidence qu'à ce chapitre ce sont les placards publicitaires qui nous fournissent la plus abondante et la plus constante information, mais il $y$ manquera toujours la garantie d'objectivité fournie par les professionnels de l'information.

4) Enfin, il y a le lecteur qui se passionne pour l'information touristique parce qu'il vit du tourisme. Assez curieusement, ce lecteur devra chercher sa pitance ailleurs que dans les pages touristiques pour savoir sil le tourisme se porte bien au Québec. pour apprendre que le gouvernement s'ap= prête â prendre telle décision politique ou à lancer tel nouvel programme d'aide á l'entreprise et ou aux gens défavorisés... Mais, enfin, les spécialistes de ces questions trouvent un écho à leurs préoccupations dans les pages politiques ou économiques, sauf que... Sauf que je ne détesterais pas avoir parfois le point de vue éclairé d'un journaliste côtoyant, à longueur d'année, des experts érangers du tourisme pour mettre par exemple, en juste perspective le succès ou l'échec d'une saison touristique chez nous.

En somme, en dépit des ressources assez importantes consacrées à l'information touristique dans nos grands quotidiens, il paraît évident que nous sommes encore loin de nous approcher de ce qui est, à mon avis, un modèle dans le monde, le New York Times, où la section Travel compte de nombreux chroniqueurs à quil l'on demande de voyager beaucoup et d'écrire peu mais, en revanche, de consacrer le plus clair de leur temps à la réécriture des textes rédigés par des dizaines de personnalités (écrivains, businessmen, artistes, etc.) qui racontent autant d'expériences de voyages différentes, ainsi qu'à la préparation de réponses rigoureusement exactes pour les chroniques de courriers de lecteurs. Et puis, au fait, les vacances et les voyages étant des expériences tellement personnelles, pourquoi n'ouvririons-nous pas les pages touristiques de nos grands journaux québécois à des dizaines de lecteurs-vacanciers-voyageurs d'ici? Plus nous serions de fous à y écrire, mieux tout le monde serait informé. $f$ 